\title{
Biometrics : Analysis of Fingerprint using Hybrid Transformations
}

\author{
Amanpreet Kaur \\ EECE Department \\ The NORTHCAP \\ UNIVERSITY \\ Gurgaon, India
}

\author{
Rekha Vig, PhD \\ EECE Department \\ The NORTHCAP \\ UNIVERSITY \\ Gurgaon, India
}

\author{
Swaran Ahuja, PhD \\ EECE Department \\ The NORTHCAP \\ UNIVERSITY Gurgaon, India
}

\begin{abstract}
A biometric system is a secured recognition system that is used for the establishment of the personal identification of the individuals using their biometrics which are unique features and make the system more authentic. Our aim, here is to build such a system which gives more accurate confirmation of the individual identities. In this paper, we have used one biometric trait i.e. fingerprint for identification. The features of fingerprints have been extracted using different combinations of existing transforms generated by the Kroneckar product of two transforms at a time to form hybrid transform. The existing orthogonal transforms which are used here are DCT, DFT, Walsh, Haar and Kekre. By generating the energy matrix and extracting the features by varying the energy threshold from $95 \%$ to $99.99 \%$ efficiency of the fingerprint biometric system obtained is up to $94.64 \%$ with the hybrid transform (DCT and Walsh combination).
\end{abstract}

\section{Keywords}

Biometrics; Recognition; Transformations; Wavelets; Kroenecker product; Hybrid transform; Fingerprint.

\section{INTRODUCTION}

Image processing is having the significant role in the digital system environment to provide the information transition based on the physiological or behavioral analysis. The basic image processing activity is the recognition system. A biometric system is essentially run by capturing the biometric data from an individual person, extracting their features from the data that has been acquired and comparing with the template which is already present in the form of dataset in database. The biometrics is divided into two main types which are based on identification and verification of the traits[3].

a) Behavioral Biometrics: These biometrics are based on the behavior and mood of the person and which doesn't remains same throughout life, for example: signature, voice, gait, keystroke, etc.

b) Physiological Biometrics: These biometrics are based on the physical body parts of a person which remains same throughout life and are unique, for example: fingerprints, iris, palm prints, face, retina, DNA, etc.

Biometric features must have these characteristics listed below for a valid biometric trait

a) Universality: Every individual must possess traits.

b) Distinctiveness: Every individual have unique trait.

c) Permanence: The trait that is sufficiently invariant. d) Collectability: The trait which is measurable.

A biometric system have two modes: verification and identification mode.[1]

a) In verification mode the person's identity is being compared with the existing acquired dataset which has its own biometric template that is lying in the database eg. Passwords /PIN's. The main aim is to prevent the multiple people to use the same identity.

b) In identification mode the system compares the identity with the many other templates in the database. It tells whether the right person has come or not.
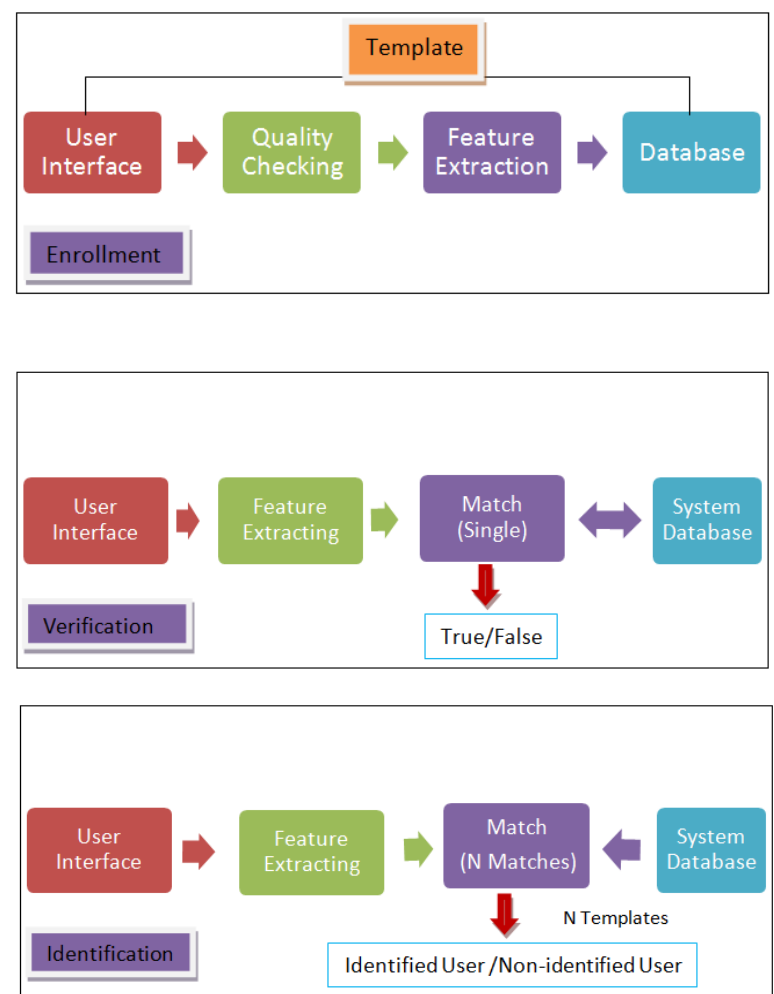

Figure1: Different modes a) Enrollment b) Verification c) Identification

In this paper we have analysed the performance of the hybrid transforms on fingerprint based recognition system. The hybrid transform is a combination of properties of two different transforms which are combined using kroneckar product of these transforms. Then the energy threshold values 
have been varied for all the combinations and efficiency is computed based on matching of the features with the database.

This paper is divided into following sections: Section II describes the related work and transformations required for the generation of hybrid transform. Section III describes the proposed methodology for the generation of energy map and feature generation of fingerprint images. Section IV describes the results we obtained and Section $\mathrm{V}$ concludes the results of the paper.

\section{LITERATURE SURVEY AND TRANSFORMATIONS REQUIRED}

A fingerprint is an impression of the friction ridges and minutiae of the finger $[9,10]$.Fingerprint matching techniques are minutiae-based and correlation-based. A new fingerprint matching algorithm has been proposed which is based on Hough transform to compute the similarities by considering both minutiae and orientation field information. Further, a fusion technique has been used which shows improved results and more accuracy [2]. In [6] proposed a fingerprint verification system which is based on minutiae extraction but due to poor quality impressions some problem lies .Some novel changes like minutia marking, segmentation using morphological operations, methods of false minutiae removal improved thinning, etc. [7]An online fingerprint identification system with a fast and a hashing distortion tolerant method. Existing authentication systems are based on passwords or tokens based which can either use magnetic cards and the passports which contains security risks due to Social attacks. Biometrics such as fingerprints, iris, palm print and voice offers more reliable authentication. However, it is difficult to design a fast and accurate biometric recognition because of presence of large databases and the complicated systems. In this paper, they present a new scheme that is able to speed up the system and improves accuracy as well. [8] Proposed a Pores and Ridges: Fingerprint Matching Using Level 3 Features. Level 1 is pattern, Level 2 is minutiae points and Level 3 is pores and ridge shape and out of that reliably they have extracted Level 3 features, AFIS employ only Level 1 and Level 2 features. This results does not improve the matching performance with the increase in the scan resolution.

In [11], a system was proposed which use fingerprint minutiae matching algorithm that was hasty, precise and appropriate for the real time recognition system. [12] They proposed a new transform i.e. discrete wavelet packet transform which is used for fingerprint matching and based on feature extraction. This transform is applied only on small area of fingerprint image. It gives the improved performance over the DWT, Gabor filter and minutiae based method. They proposed an approach which is used for embedding two watermarks into fingerprint images using the DCT algorithm. The existing scheme shows a high PSNR value [13]. The author proposed an advanced method that are based on fractional fingerprint for recognition system works micro-features, local analysis of fingerprints and bifurcations [5].

The transforms are used to extract the information from images. These are different types of orthogonal transforms and wavelets are there which provides frequency domain coefficients of the image. There exists a large number of verification and identification system which are based on feature extraction using various transforms such as Fourier transform [4]. All these transforms have the tendency to concise the energy in fewer components if we neglect the energy in higher frequency components and these coefficients can be used in biometric identification to represent the various features. There exist various mathematical tools which are being used for extracting information from images. The spectral component $\mathrm{n}$ a given time interval can be determined using these mathematical tools.

Identification can be performed in two domains:

Spatial domain: In Spatial domain features of fingerprints can be analyzed like ridges and valleys.

Transform domain: In Transform domain there is a transform matrix for frequency domain that can be any transform like DFT, DCT, DST, Walsh transform, Haar transform etc[4].

$$
\mathrm{I}=\mathrm{T}_{\mathrm{d}} * \mathrm{im} * \mathrm{~T}_{\mathrm{d}}^{\mathrm{t}} \ldots
$$

Where im- spatial domain image, Td -transform matrix \& Tdt -the transform of Td.

Transform Domain Analysis: The various combinations of five transforms are used here. These five transforms are:

Discrete Cosine Transform (DCT): DCT is considered as the adaptive transformation approach to extract the image features. In this DCT is applied on the window block of the available image set as well as on input image.

$$
\mathrm{F}(u)=\left(\frac{2}{\mathrm{~N}}\right)^{\frac{1}{2}} \sum_{i=0}^{N-1} \mathrm{~A}(\mathrm{i}) \operatorname{Cos}\left[\frac{\pi \mathrm{u}}{2 \mathrm{~N}}(2 \mathrm{i}+1)\right] \mathrm{f}(\mathrm{i})
$$

where $\mathrm{i}$ is specific position.

Kekre Transform: The matrix size can be used on images of any size and all upper diagonal elements are one and diagonal elements are also one and also the lower diagonal elements are zero except the elements just below the diagonal. Kekre transform matrix is given by:

$$
K_{x y}=\left\{\begin{array}{cc}
1 & x \leq y \\
-N+(x-1) & x=y+1 \\
0 & x>y+1
\end{array}\right.
$$

DFT Transform: The discrete Fourier transform or DFT is equivalent to continuous FT for the transform that deals with a finite sequence of data or discrete-time signal with discrete number of frequencies.

Walsh Transform: As any orthogonal (unitary) matrix can be used to define a discrete orthogonal (unitary) transform, Walsh transform is non-sinusoidal that decomposes into a set of orthogonal two values +1 and -1 rectangular waveforms called Walsh functions. It has no multipliers and is real because of the amplitude .An important property is sequency.

Haar Wavelet: The wavelet analysis is performed using a prototype function $\emptyset(\mathrm{x})$ i.e. mother wave. Temporal analysis is done with a contracted daughter wavelet, which is high frequency version of mother wavelet, while with a dilated low frequency version ;frequency analysis is performed. That signal is $f(\mathbf{x})$ can be analyzed using wavelets by creating a variable scale mathematical functions.

$$
\emptyset_{(\mathrm{sf})}(\mathrm{x})=2^{-\frac{s}{2}} \emptyset\left(2^{-\mathrm{s}} \mathrm{x}-1\right)
$$

Where $\mathrm{s}$-scale indexes and \& 1 -dilate the position of the mother function to generate wavelets. Haar Wavelet is most commonly used in recognition algorithms. The input signal is decomposed into vertical, diagonal,horizontal and approximate ccoefficients using the wavelet transformation and the higher level coefficients for are used to reduce the 
space complexity and is used to discard the redundant information.

Kroneckar Product : Kroneckar Product of two matrices P and $\mathrm{Q}$ is given as

$$
\mathrm{K}=\mathrm{P} \otimes \mathrm{Q}=\left[\mathrm{p}_{\mathrm{ij}} \mathrm{Q}\right] .
$$

Where $\mathrm{K}$ is $\mathrm{mn} * \mathrm{mn}, \mathrm{P}$ is $\mathrm{m} * \mathrm{~m}$ and $\mathrm{Q}$ is $\mathrm{n} * \mathrm{n}$.

$\mathrm{K}$ matrix is given by

$$
[K]=\left[\begin{array}{ccccc}
p_{11} Q & p_{12} Q & \cdots & \cdots & p_{1 m} Q \\
p_{21} Q & p_{22} Q & \cdots & \cdots & p_{2 m} Q \\
\vdots & \vdots & \vdots & \vdots & \vdots \\
p_{m 1} Q & p_{m 2} Q & \cdots & \cdots & p_{m m} Q
\end{array}\right]
$$

Both $\mathrm{P}$ and $\mathrm{Q}$ have to be orthogonal for $\mathrm{K}$ to be orthogonal.

Hybrid Transform: Hybrid transform is generated using Kroneckar product of two different transforms by combining the properties of both the transforms. Here, we have used the different combinations of DCT, Walsh, Kekre, Haar and DFT transforms. In this $\mathrm{P}$ and $\mathrm{Q}$ matrices are combination of two $\left(16^{*} 16\right)$ matrices which have two different transforms combinations like DCT and Walsh, DCT and Haar, Haar and Walsh, Haar and Kekre, DCT and Kekre, Walsh and Kekre and DCT and DFT.[4]

\section{PROPOSED METHODOLOGY}

In this method, Fingerprint images have been saved in the database which are scaled into $256 * 256$ size. Transform matrix is formed by the kroneckar product of two transforms as explained earlier and then after the transformation energy matrix is generated.

a) Generation of the energy matrix: The image database consists of extracted fingerprint images of size $256 * 256$.Each database image is firstly transformed in transform domain using equation (i) and then energy matrix is generated for each of the images in the database.

b) Generation of feature vector map: The energy matrix of size $256 * 256$ is divided into 4096 blocks of $4 * 4$ size each and the mean is computed for each block which generates a $64 * 64$ energy block matrix.

c) Feature Selection and Storing: Energy map is used for feature selection. These blocs are selected based on the threshold energy value of the energy matrix.

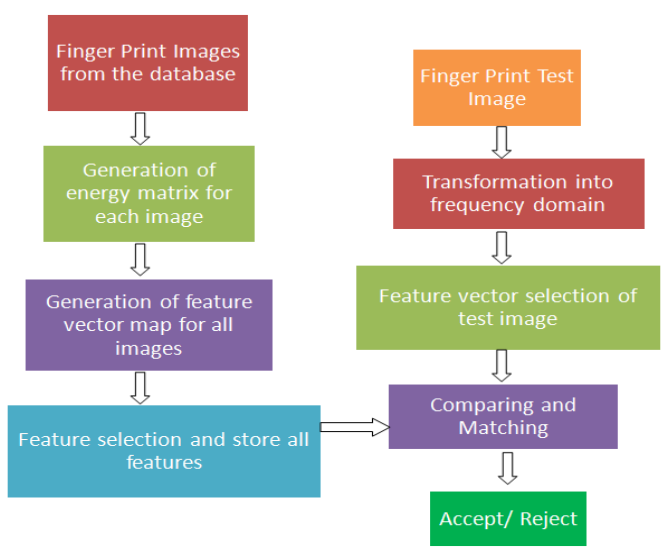

Figure 4: Proposed Methodology d) Transformation: The test image is transformed into frequency domain using different hybrid transforms.

e) Feature Selection of test image: Feature selection would be same as explained for the database images.

f) Comparing and Matching: These features will be arranged in the descending order of their energy values and then taking the higher energy coefficients and finding the match with the database and hence efficiency is computed based on comparison.

g) Acceptance /Rejection: The accuracy of the system is based on matching result whether it is accepted or rejected.

\section{RESULTS}

The database consists of 168 images of fingerprints .One image is considered as the test image and is scaled to $(256 * 256)$ size. The energy threshold is varied from $95 \%$ to $99.99 \%$ for analysing the performance of the hybrid transformation on the fingerprint images. The results have been shown below for all the combinations used.

Table 1: Results For The Comaprison Of Different

\begin{tabular}{|c|c|c|c|c|}
\hline Threshold & $\begin{array}{l}\text { No. of } \\
\text { Features }\end{array}$ & $\begin{array}{l}\text { Effective } \\
\text { matches }\end{array}$ & Efficiency & Transformation \\
\hline 0.9999 & 4059 & 159 & 94.6429 & DCT and Walsh \\
\hline 0.99 & 3005 & 158 & 94.0476 & \\
\hline 0.98 & 2622 & 158 & 94.0476 & \\
\hline 0.97 & 2288 & 157 & 93.4524 & \\
\hline 0.96 & 2051 & 157 & 93.4524 & \\
\hline 0.95 & 1875 & 157 & 93.4524 & \\
\hline 0.9999 & 4064 & 158 & 94.0476 & DCT and Haar \\
\hline 0.99 & 3006 & 158 & 94.0476 & \\
\hline 0.98 & 2627 & 158 & 94.0476 & \\
\hline 0.97 & 2319 & 158 & 94.0476 & \\
\hline 0.96 & 2055 & 158 & 94.0476 & \\
\hline 0.95 & 1879 & 158 & 94.0476 & \\
\hline 0.9999 & 3840 & 156 & 92.8571 & Haar and Walsh \\
\hline 0.99 & 2534 & 156 & 92.8571 & \\
\hline 0.98 & 2124 & 155 & 92.2619 & \\
\hline 0.97 & 1857 & 155 & 92.2619 & \\
\hline 0.96 & 1651 & 155 & 92.2619 & \\
\hline 0.95 & 1487 & 156 & 92.8571 & \\
\hline 0.9999 & 3927 & 152 & 90.4762 & Haar and Kekre \\
\hline 0.99 & 3287 & 152 & 90.4762 & \\
\hline 0.98 & 3065 & 152 & 90.4762 & \\
\hline 0.97 & 2895 & 152 & 90.4762 & \\
\hline 0.96 & 2753 & 152 & 90.4762 & \\
\hline 0.95 & 2627 & 152 & 90.4762 & \\
\hline 0.9999 & 4090 & 152 & 90.4762 & DCT and Kekre \\
\hline 0.99 & 3859 & 152 & 90.4762 & \\
\hline 0.98 & 3693 & 151 & 89.881 & \\
\hline
\end{tabular}
Combinations Of Hybrid Transforms 


\begin{tabular}{|c|c|c|c|c|}
\hline 0.97 & 3556 & 151 & 89.881 & \\
\hline 0.96 & 3427 & 152 & 90.4762 & \\
\hline 0.95 & 3304 & 153 & 91.0714 & \\
\hline 0.9999 & 4093 & 150 & 89.2857 & Walsh and Kekre \\
\hline 0.99 & 3883 & 150 & 89.2857 & \\
\hline 0.98 & 3731 & 150 & 89.2857 & \\
\hline 0.97 & 3599 & 150 & 89.2857 & \\
\hline 0.96 & 3473 & 150 & 89.2857 & \\
\hline 0.95 & 3353 & 151 & 89.881 & \\
\hline 0.9999 & 4096 & 151 & 89.881 & DCT and DFT \\
\hline 0.99 & 4096 & 151 & 89.881 & \\
\hline 0.98 & 4096 & 151 & 89.881 & \\
\hline 0.97 & 4096 & 151 & 89.881 & \\
\hline 0.96 & 4096 & 151 & 89.881 & \\
\hline 0.95 & 4096 & 151 & 89.881 & \\
\hline
\end{tabular}

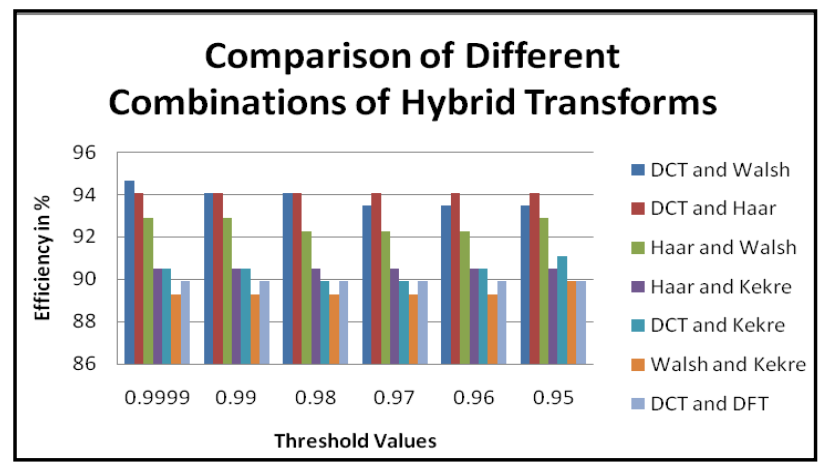

\section{CONCLUSIONS}

In this paper we have used hybrid transforms to analyse the performance of fingerprint based recognition system. The kroneckar product of DCT and Walsh transforms will give the maximum efficiency $94.6429 \%$ at the threshold value $99.99 \%$ which implies this combination is suitable for fingerprint recognition systems as this will match much more effectively than other combinations.

\section{REFERENCES}

[1] A. K. Jain, A. Ross, and S. Prabhakar, "An introduction to biometric recognition," IEEE Trans. on Circuits and Systems for Video Technology, vol. 14, pp. 4-20, Jan 2004.

[2] A. A. Paulino, J. Feng, and A. K. Jain, "Latent fingerprint matching using descriptor-based Hough transform," in Int'l Joint Conf. on Biometrics, October 2011, pp. 1-7.

[3] Arun Ross and Anil K. Jain, "Multimodal Biometrics: An Overview" Proc. of 12th European Signal Processing Conference (EUSIPCO), (Vienna, Austria), pp. 12211224, September 2004.

[4] Kekre, H. B., Rekha Vig, Saurabh Bisani, Tanuja Sarode, Pranay Arya, and Aashita Irani. "Identification of multispectral palmprints using energy compaction by Hybrid wavelet", 2012 5th IAPR International Conference on Biometrics (ICB), 2012.

[5] V. Conti, G. Vitello and F. Sorbello, S. Vitabile, "An Advanced Technique for User Identification using Partial Fingerprint", 2013 Seventh International Conference on Complex, Intelligent, and Software Intensive Systems.

[6] Manvjeet Kaur, Mukhwinder Singh, Akshay Girdhar, and Parvinder S. Sandhu, "Fingerprint Verification System using Minutiae Extraction Technique." World academy of Science, Engineering and Technology, page no. 46, 2008.

[7] Hoi Le, The Duy Bui, "Online fingerprint identification with a fast and distortion tolerant hashing." Journal of Information Assurance and Security 4 page no. 117-123, 2009.

[8] Anil Jain, Yi Chen, and Meltem Demirkus, "Pores and Ridges: Fingerprint Matching Using Level 3 Features." Pattern recognition letters, page no. 2221-2224, 2004.

[9] A. Ross, S. Dass, and A.K. Jain, "A deformable model for fingerprint matching", Journal of pattern Recognition, Elsevier, Vol. 38, no1.

[10] T. Matsumoto, H. Hoshinok. Yamada and Hasino, "Impact of artificial gummy fingers on fingerprint systemt", In Proc. of SPIE, volume 4677.

[11] Jie Y, Fang Y, Renjie Z., Qifa S. fingerprint minutae matching algoritham for real time system. Pattern Recogn 2006; 39:143-6

[12] Sangita Bharkad, Manesh Kokare, "Fingerprint Matching using Discreet Wavelet Packet Transform", 2013 3rd IEEE International Advance Computing Conference (IACC).

[13] Mohammed Alkhathami, Fengling Han and Ron Van Schyndel, "Fingerprint Image Protection Using Two Watermarks Without Corrupting Minutiae", 2013 IEEE 8th Conference on Industrial Electronics and Applications (ICIEA). 